\title{
On Spacetime Duality and Bounce Cosmology of a Dual Universe
}

\author{
Mohammed. B. Al-Fadhli ${ }^{*}$ \\ ${ }^{1}$ College of Science, University of Lincoln, Lincoln, LN6 7TS, UK. \\ *Correspondence: malfadhli@lincoln.ac.uk; mo.fadhli7@gmail.com
}

\begin{abstract}
Precise astronomical measurements of the fine structure constant and universe expansion rate have revealed that they vary over specific directions, demonstrating an anisotropic universe. The curvature in complex spacetime can be interpreted as spatial warping evolution along with its travel through the imaginary time dimension. Complex spacetime worldlines of the universe spatial factor evolution through imaginary time are utilised to model universe anisotropy. The worldlines of a positively curved universe revealed both positive and negative solutions, which imply that matter and antimatter could be evolving in opposite directions as distinct sides of the universe, theoretically corroborating the axis of the cosmic microwave background and observed anisotropy. The model indicates that a nascent hyperbolic expansion is followed by a first phase of decelerating spatial expansion during the first $\sim 9$ Gyr, and then, a second phase of accelerating expansion. The model potentially resolves the tension in Hubble parameter measurements, with a predicted density at the phase transition of $\Omega=\sim 1.12>1$. In addition, it predicts a final time-reversal phase of rapid spatial contraction leading to the Big Crunch, signalling a cyclic universe. On spacetime quantum duality, the simulations of the spacetime continuum flux through its travel along with its predicted worldlines demonstrated the fast-orbital speed of stars resulting from an external momentum exerted on galaxies via the spatial curvature through the imaginary time dimension. These findings indicate that antimatter could exist as a distinct side, which influences the universe evolution; physically explaining the effects attributed to dark matter and dark energy.
\end{abstract}

Keywords: Accelerated expansion, fast-rotation, flatness, parallel universe, duality, antimatter

\section{INTRODUCTION}

Advances in cosmology and astronomical observations over the last two decades have revealed many inconsistencies with our traditional view of the universe. Precise astronomical measurements of the fine structure constant over multiple directions in several studies revealed that it varies through two directions. The variation occurs both with time and across a specific axis [1-2]. Additionally, the expanding rate of the universe has been observed to vary depending on direction. These consistent observations indicate a strong anisotropy at about five-sigma confidence level [3].

Modelling universe anisotropy utilising complex spacetime worldlines of the universe spatial factor evolution through imaginary time might demonstrate the universe evolutionary topology and provide physical reasoning for the detected anisotropy and other unsolved universe problems.

Additionally, bounce cosmology could provide agreement with anisotropy observations in the cosmic microwave background (CMB) such as the potential early universe positive curvature [4], and the $\mathrm{CMB}$ axis and dipole anisotropy. The non-singular Big Bounce theory assumes that primordial substance was concentrated from a previous collapsed universe, with the universe experiencing continuous expansions and contractions, where the initial spatial factor is greater than zero $[5,6]$. However, another version of the theory is postulated that a Bounce Bang of the primordial substance at thermal equilibrium produced a hot and dense early universe, where matter and antimatter could have been separated by electromagnetic fields $[7,8]$ and propelled in opposite directions in support of the CMB dipole. Sings of this primordial Bang might have been observed as the associated noise surrounding the measured gravitational waves [9] as a cosmic gravitational background (CGB). 
Recently, inconsistencies with the current standard lambda cold dark matter model ( $\Lambda \mathrm{CDM})$ have emerged in new astronomical observations [4,10]. The recent Planck Legacy 2018 (PL18) release indicated the existence of an enhanced lensing amplitude in the $\mathrm{CMB}$ from the early universe, which is higher than predicted by the $\Lambda \mathrm{CDM}$ [11]. This endorses the existence of a positive curvature with a confidence level greater than $99 \%$ [4]. Besides, the observed gravitational lensing by substructures of 11 galaxy clusters is an order of magnitude greater than that predicted by the $\Lambda$ CDM [12]. Riess, in 2020, found that the expansion of the universe is faster than that estimated by the $\Lambda C D M$, with the disagreement between several independent measurements taken from the early universe using the Planck datasets based on $\mathrm{CMB}$ and present universe using the type Ia supernovae distance-redshift method [10,13-15] being four to six standard deviations [16]. Further, the speed of expansion has been observed to vary depending on the direction [3]. Thus, emerging evidence supports a growing need for a profound adjustment to the $\Lambda \mathrm{CDM}$ or new physics $[17,18]$.

In this study, complex spacetime worldlines were utilised to model the universe anisotropy. A closed finite universe of non-singular Bounce Bang creation was adopted as it could be aligned with quantum entanglement, where the existing energy is preserved indefinitely. Cosmic conservation therefore ensures the total spin of a pair of particles are always conserved regardless of their locations [19]. Further, the closed universe could provide agreement with CMB anisotropy observations [20]. The paper is organised as follows. In Section 2, the model is derived with counting for the universal curvatures and without a cosmological constant as its predicted value contradicts the quantum field theory [21]. Sections 3, 4, and 5 discuss the evolution of spacetime worldlines, simulation of spacetime curvatures, and the expansion rate, respectively. Section 6 demonstrates the fast-orbital star speed. Section 7 summarises the conclusions. Finally, Section 8 suggests future developments of this work.

\section{MATHEMATICAL MODEL}

The fine-structure constant has been detected to vary through space and time [1-3], where the measured values of speed of light in vacuum $c_{\eta}$, Newton's gravitational 'constant' $G_{\eta}$, Planck force $F_{p, \eta}$, etc, could vary over the universe evolution stages, as a function of the conformal time $\eta$. As an alternative, spacetime is deemed to have a dual quantum nature in that it curves according to general relativity as waves and travel at speed of light as quantum particles. Spacetime modulus of elasticity $E_{D}=\left(\right.$ stress/strain) in $\left(\frac{N}{m^{2}}\right)$ is considered as a fundamental constant of nature; it is written in terms the stress energy-momentum tensor $T_{v}^{u}$ and its trace $T$ divided over their resulting change in curvature signified by Ricci curvature tensor $R_{v}^{u}$ with respect to the conformal universal scalar curvature $R_{\eta}$

$$
E_{D}=\frac{T_{v}^{u}-\frac{1}{2} T \delta_{v}^{u}}{R_{v}^{u} / R_{\eta}},
$$

where $\delta_{v}^{u}$ is Kronecker delta [22]. The Einstein-Hilbert action is then written

$$
S=\int\left[E_{D} \frac{R}{2 R_{\eta}}+L\right] \sqrt{-g} d^{4} x,
$$

here $R=R_{u v} g^{u v}$ is the scalar curvature over space and imaginary time $\tau, R_{\eta}$ is the scalar curvature over space and conformal time $\eta, L$ denotes the Lagrangian of matter/antimatter, $g=\operatorname{det} g_{u v}$ is the determinant of the metric tensor $g_{u v}$. Using the principle of least action, $S$ yields an extended form of Einstein's field equations with incorporating the conformal concurrent universal curvature

$$
\frac{R_{u v}}{R_{\eta}}-\frac{R}{2 R_{\eta}} g_{u v}=\frac{T_{u v}}{E_{D}} .
$$

Using the Newtonian limit, $E_{D}=4.815 \times 10^{42} \mathrm{~N} / \mathrm{m}^{2}$ given by $F_{p, \eta}$ over $8 \pi r_{\eta}{ }^{2}$. Here $r_{\eta}{ }^{2}$ denotes the inverse of scalar curvature $R_{\eta}$, which has almost no local effect due to current spacetime flatness $r_{c}^{2} \cong 1$. Yet, it is significant in the early universe due to the existence of a positive curvature [4] where $r_{\eta}{ }^{2}>1$. The curvature tensor resulting from traveling in conformal time was excluded to simplify the extended field equations, where the curvature in $\tau$ is much greater than the curvature in $\eta$. 
The initial universal curvature $r_{0}{ }^{2}$ can be integrated into the Friedmann-Lemaître-RobertsonWalker (FLRW) metric model, which characterises $g_{u v}[23,24]$, as the inverse of the initial scalar curvature $R_{0}$. Thus, the enhanced isotropic spherical coordinates are

$$
d s^{2}=-c_{\eta}{ }^{2} d \tau^{2}-a^{2}(-i \tau)\left(\frac{d r^{2}}{1-k r^{2} / r_{0}{ }^{2}}+r^{2} d \theta^{2}+r^{2} \sin ^{2} \theta d \phi^{2}\right),
$$

where $d s$ is the four-dimensional complex spacetime interval in polar coordinates, $a$ is the spatial factor and $k$ is the type of space curvature. For a closed, open, and flat universe, $k=1,-1$, and 0 ; while $r_{0}^{2}=1,>1$, and $<1$ for flat, positive, and negative initial space curvatures respectively.

The Ricci scalar curvature and Ricci curvature tensor components are solved using Christoffel symbols for the enhanced $g_{u v}$ as follows

$$
\begin{gathered}
R=\frac{6\left(a \ddot{a}+\dot{a}^{2}+k c_{\eta}{ }^{2} / r_{0}{ }^{2}\right)}{-c_{\eta}{ }^{2} a^{2}}, \quad R_{0}^{0}=3 \frac{-\ddot{a}}{a}, \quad R_{1}^{1}=\frac{\ddot{a}+2 \dot{a}^{2}+2 k c_{\eta}{ }^{2} / r_{0}{ }^{2}}{c_{\eta}{ }^{2}\left(1-k r^{2} / r_{0}{ }^{2}\right)}, \\
R_{2}^{2}=\frac{r^{2}\left(a \ddot{a}+2 \dot{a}^{2}+2 k c_{\eta}{ }^{2} / r_{0}{ }^{2}\right)}{c_{\eta}}, \quad R_{3}^{3}=\frac{r^{2} \sin ^{2} \theta\left(a \ddot{a}+2 \dot{a}^{2}+2 k c_{\eta}{ }^{2} / r_{0}{ }^{2}\right)}{c_{\eta}{ }^{2}} .
\end{gathered}
$$

In addition, by solving the field equations in Eq. (3) for a perfect fluid given by $T_{v}^{u}=\left(\rho+\frac{P}{c_{\eta}{ }^{2}}\right) u^{u} u_{v}+$ $P \delta_{v}^{u}[22,25]$ and applying Eqs. $(5,6)$, Friedmann equations in terms of the initial and conformal universal curvatures are obtained

$$
\begin{gathered}
H^{2} \equiv \frac{\dot{a}^{2}}{a^{2}}=\frac{\rho c_{\eta}{ }^{4}}{3 E_{D} r_{\eta}{ }^{2}}-\frac{k c_{\eta}{ }^{2}}{a^{2} r_{0}{ }^{2}}, \\
\dot{H} \equiv \frac{\ddot{a}}{a}=-\frac{c_{\eta}{ }^{4}}{6 E_{D} r_{\eta}{ }^{2}}\left(\rho+3 \frac{P}{c_{\eta}{ }^{2}}\right) .
\end{gathered}
$$

where $H, P$, and $\rho$ are the Hubble parameter, pressure, and density respectively. The Friedman equations can be solved over the entire imaginary conformal time of current universe life cycle, where $k=1$ by the definition of the FLRW metric for a positively curved universe. By rewriting Eq. (7) in terms of the imaginary conformal time in its parametric form $d \eta=\frac{d \tau}{i a}$ and the derivative of the spatial factor with regards to the imaginary time $\dot{a}=i \frac{d a}{d \tau}$ as

$$
\int_{\eta_{0}}^{\eta} d \eta=\oint\left(\frac{c_{\eta}{ }^{4} \rho_{0} a_{0}{ }^{3}}{3 E_{D} r_{\eta}{ }^{2}} a-\frac{c_{\eta}{ }^{2}}{r_{0}{ }^{2}} a^{2}\right)^{-\frac{1}{2}} d a
$$

where $\rho=\frac{\rho_{0} a_{0}{ }^{3}}{a^{3}}$ [26], $a_{0}$ is the spatial factor at the corresponding imaginary time $\tau_{0}$ and density $\rho_{0}$. By integrating, the evolution of the spatial factor is obtained

$$
a(\eta)=\frac{\rho_{0} r_{0}^{2} a_{0}^{3}}{12 E_{D}} \frac{c_{\eta}{ }^{2}}{r_{\eta}^{2}}\left(1-\cos \frac{\eta}{r_{0}}\right) .
$$

Additionally, the evolution of imaginary time $\tau(\eta)$ can be obtained by integrating the length of the spatial factor contour of one universe life cycle over its expansion speed $H_{\eta}$, initiating at imaginary time $\tau_{0}$ with the corresponding spatial factor $a_{0}$. Therefore, by rewriting Eq. (10) in terms of the Hubble parameter by its definition at $\tau_{0}$ as $d \tau=i \frac{d a}{H a_{0}}$ as follows

$$
\int_{\tau_{0}}^{\tau} d \tau=i \int_{\eta_{0}}^{\eta} \frac{\rho_{0} r_{0}^{2} a_{0}^{2}}{12 E_{D}} \frac{c_{\eta}^{2}}{H_{\eta} r_{\eta}^{2}}\left(1-\cos \frac{\eta}{r_{0}}\right) d \eta
$$

By performing the integration while approximating $\frac{d c_{\eta}}{d \eta}=\frac{d r_{\eta}}{d \eta}=\frac{H_{\eta}}{d \eta} \cong 0$ as they are $\ll \frac{d \tau}{d \eta}$ as

$$
\tau(\eta)=i \frac{\rho_{0} r_{0}^{2} a_{0}^{2}}{12 E_{D}} \frac{c_{\eta}^{2}}{H_{\eta} r_{\eta}^{2}}\left(\eta-\sin \frac{\eta}{r_{0}}\right)+\tau_{0}
$$

where $\tau_{0}$ denotes the reference imaginary time for the temporal evolution. 
According to the non-singular Bounce Bang, the initial density of the primordial substance of the previous collapsed universe $\rho_{0}$ should have the highest value; then, it should decrease due to the increasing universe size, where $a_{0}$ and $\tau_{0}$ are the corresponding initial spatial factor and imaginary time, respectively. Therefore, according to the conservation of energy, the divergence of the energymomentum tensor vanishes at $\Delta_{v} T^{u v}$, which yields $\frac{\dot{a}}{\mathrm{a}} T_{u}^{u}+3 \frac{\dot{a}}{\mathrm{a}} \rho-i \frac{\partial \rho}{\partial \tau}=0$ and $3(\rho+P) \frac{\dot{a}}{\mathrm{a}}-i \frac{\partial \rho}{\partial \tau}=0$. By combining these outcomes, integrating, and then, submitting the spatial rate in Eq. (10) to their outcome, the density evolution is obtained

$$
\rho_{\eta}=\frac{36 E_{D}{ }^{3} D_{0}}{\rho_{0}{ }^{3} r_{0}{ }^{6} a_{0}{ }^{9}} \frac{r_{\eta}{ }^{6}}{c_{\eta}{ }^{6}}\left(1-\cos \frac{\eta}{r_{0}}\right)^{-3}
$$

where $D_{0}$ is a constant. According to Eq. (8), the Hubble parameter is dependent on the density; thus, by substituting Eq. (13) to Eq. (8) and initiating at $\tau_{0}$ where $\dot{H}=i \frac{d^{2} a}{d \tau^{2} a_{0}} \& \ddot{a}=i \frac{d^{2} a}{d \tau^{2}}$ as

$$
\int_{H_{0}}^{H} \dot{H}=\int_{a_{0}}^{a} i \frac{d^{2} a}{d \tau^{2} a_{0}}=\int_{\eta_{0}}^{\eta}-\frac{6 E_{D}{ }^{2} D_{0}}{\rho_{0}{ }^{3} r_{0}{ }^{6} a_{0}{ }^{10}} \frac{r_{\eta}{ }^{4}}{c_{\eta}{ }^{2}}\left(1-\cos \frac{\eta}{r_{0}}\right)^{-3} d \eta,
$$

The expansion rate over the universe life cycle is then obtained by integrating

$$
H_{\eta}=\frac{3 E_{D}{ }^{2} D_{0}}{2 \rho_{0}{ }^{3} r_{0}{ }^{6} a_{0}{ }^{10}} \frac{r_{\eta}{ }^{4}}{c_{\eta}{ }^{2}}\left(\frac{1}{5} \cot ^{5} \frac{\eta}{2 r_{0}}+\frac{2}{3} \cot ^{3} \frac{\eta}{2 r_{0}}+\cot \frac{\eta}{2 r_{0}}\right)+H_{0} .
$$

where $H_{0}$ denotes the integration constant. Combining Eqs. $(10,12-15)$ in the complex plane results in the polar radius of the complex spacetime worldlines

$$
\underset{0<\eta \leq 2 \pi}{\overrightarrow{R_{L}}}(\eta)=\mp \frac{\rho_{0} r_{0}^{2} a_{0}^{3}}{12 E_{D}} \frac{c_{\eta}{ }^{2}}{r_{\eta}^{2}}\left(\left(1-\cos \frac{\eta}{r_{0}}\right)^{2}+H_{\eta}^{-2} a_{0}^{-2}\left(\eta-\sin \frac{\eta}{r_{0}}\right)^{2}\right) \frac{1}{2} e^{i \cot H_{\eta} a_{0}\left(1-\cos \frac{\eta}{r_{0}}\right)\left(\eta-\sin \frac{\eta}{r_{0}}\right)^{-1}}
$$

The positive and negative solutions of the polar radius imply that matter and antimatter are evolving in opposite directions. The $c_{\eta}$ and other parameters are evolving along the spacetime worldlines and are constant along the perpendicular directions as will be demonstrated in the next section.

\section{EVOLUTION OF SPACETIME WORLDLINES}

The integration constants of the derived model in Eq. (16) were tuned to guide the mean evolution value of the Hubble parameter at $\sim 70 \mathrm{~km} \cdot \mathrm{s}^{-1} \cdot \mathrm{Mpc}^{-1}$ and a phase transition of expansion at universe age of $\sim 9$ Gyr. Positive initial curvature is considered $r_{0}{ }^{2}=1.1$ based on CMB findings [4]. Change in $r_{\eta}{ }^{2}$ was ignored due to its dependent on small variation in $c_{\eta} \& G_{\eta}$ [1-3]. Consequently, the predicted density at the phase transition is $\Omega=\sim 1.12$ and Fig. 1 shows the predicted worldlines.

Cosmic evolution is predicted to experience three distinct phases. First, both sides of the universe expand away from the Bounce Bang. This could be due to the Bang and electromagnetic fields that separated matter and antimatter and propelled them in opposite directions, where they might be blue and red shifted according to the $\mathrm{CMB}$ dipole anisotropy. During the first phase (i.e., the first $\sim 9 \mathrm{Gyr}$ ), the gradients of the worldlines indicate the expansion is decelerating. The expansion rate is discussed in Section 5 revealing a nascent hyperbolic expansion. In the second phase, the worldlines reverse their directions, with both sides entering a state of free fall towards each other. It is conceivable that the matter and antimatter are free-falling towards each other with gravitational acceleration, causing accelerated expansion. The gradients of worldlines during this phase show an accelerated rate of expansion. These two phases can be explained using the analogy of a ball being thrown upwards, where the ball decelerates until it stops, then reverses its direction, and free falls. 
Interestingly, the model predicts a third phase of spatial contraction with a reversal-time arrow that appears after $\sim 18$ Gyr. In this phase, the universe experiences a contraction, which could be due to a high concentration of matter/antimatter on both sides, leading to the Big Crunch.

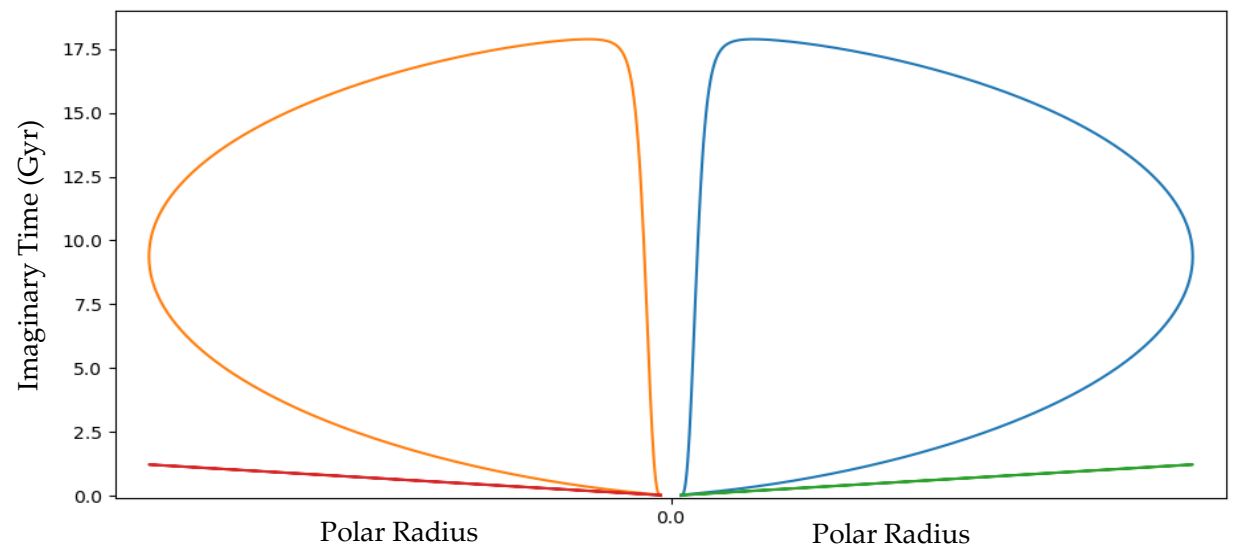

FIG. 1. Evolution of the complex spacetime worldline polar radius of the universe spatial factor through imaginary time on both sides (curves) in addition to the straight lines of light worldlines (diagram is not to scale). The evolutions are, at first, a phase of spatial expansion away from the Bounce Bang on both sides. Then, a second spatial expansion phase occurs in the reverse direction as both sides free-fall towards each other at gravitational acceleration. Finally, a third time-reversal phase of spatial contraction, leading to the Big Crunch.

A 3D spatial and 1D temporal dimension schematic of the spacetime worldline evolutions of both sides of the universe is shown in Fig. 2.

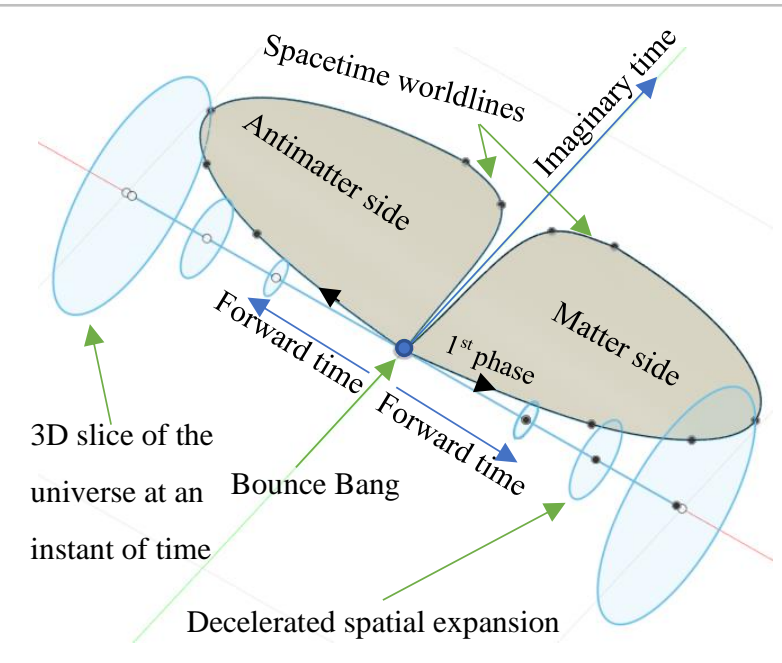

(a)

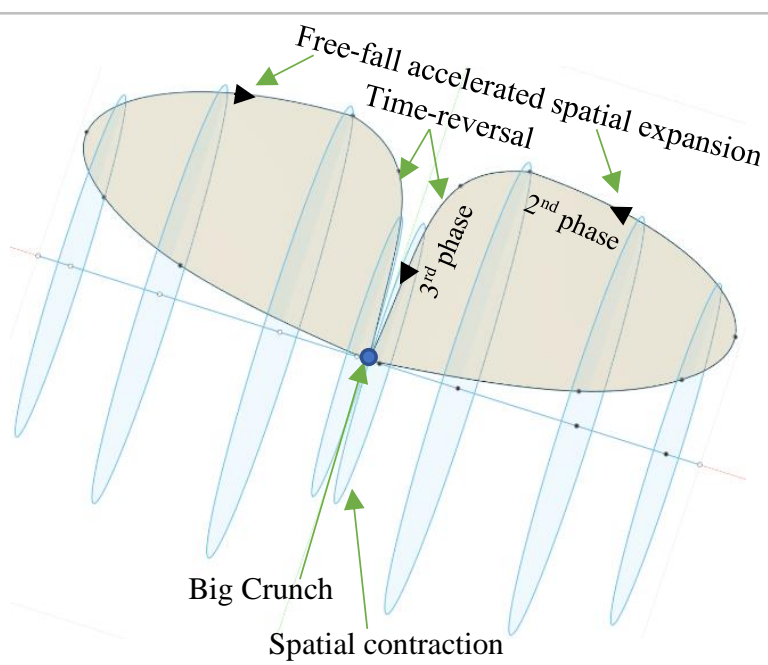

(b)

FIG. 2. Schematic in 3D spatial and 1D temporal dimensions of both sides, according to the spacetime worldline evolution. (a) In the first phase, both sides expand away from the Bounce Bang. (b) In the second stage, both sides expand in reverse directions and free-fall towards each other at gravitational acceleration. In the third phase, both sides contract, due to high concentrations of matter/antimatter, leading to the Big Crunch. Blue circles represent a 3D universe slice, which is not necessarily a simply path connected.

$c_{\eta}$ evolves along the spacetime worldlines and remain constant in the perpendicular directions, where its variation can be obtained from [1-2]. $\tau c_{\eta}$ affects each side directly due to its expansion. $c_{\eta}$ times Forward time affects the expansion of both sides due to the gravity between them. The spacetime worldlines would appear as straight lines as will be discussed in the following section. 


\section{SIMULATION OF SPACETIME CURVATURE}

The spacetime worldlines of a single side can be simulated according to the derived model with nearby spacetime worldlines corresponding to the early and present universe, as shown in Fig. 3. In the early universe, the simulation of spacetime worldline evolutions coupled with flat and positive initial curvatures are not equal at any age during the first phase, as shown in Fig. 3(a). This reveals that the spacetime is curved similar to the curved surface of a ball, which aligns with recent reports of space curvature from CMB [4]. Conversely, for the present accelerated phase of expansion in the reverse direction, the simulation of the evolution of spacetime worldlines coupled with the positive initial curvature produce a flat end or flat spacetime, as shown in Fig. 3(b).

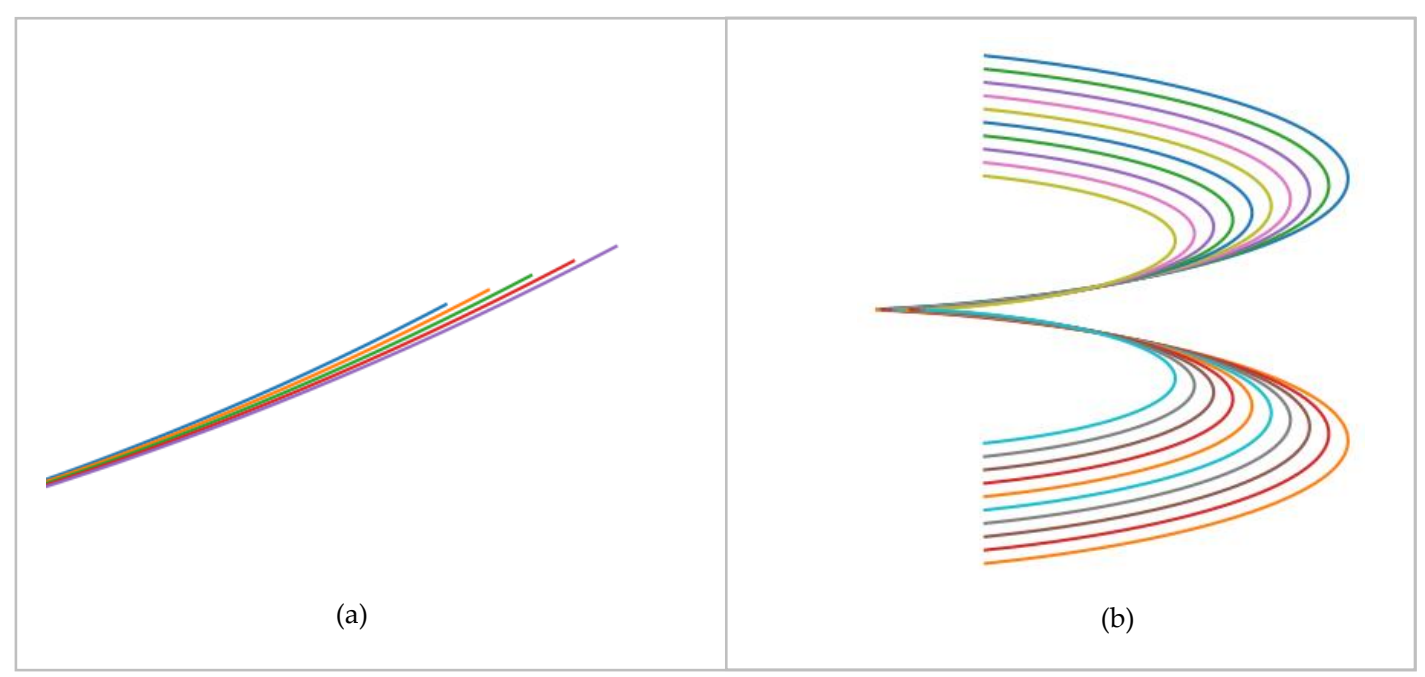

FIG. 3. Evolution of spacetime worldlines corresponding to (a) the early and (b) the present universe.

A schematic of a 2D spatial and 1D temporal dimension is shown in Fig. 4(a), while Fig. 4(b) shows an approximate apparent topology due to gravitational lensing.

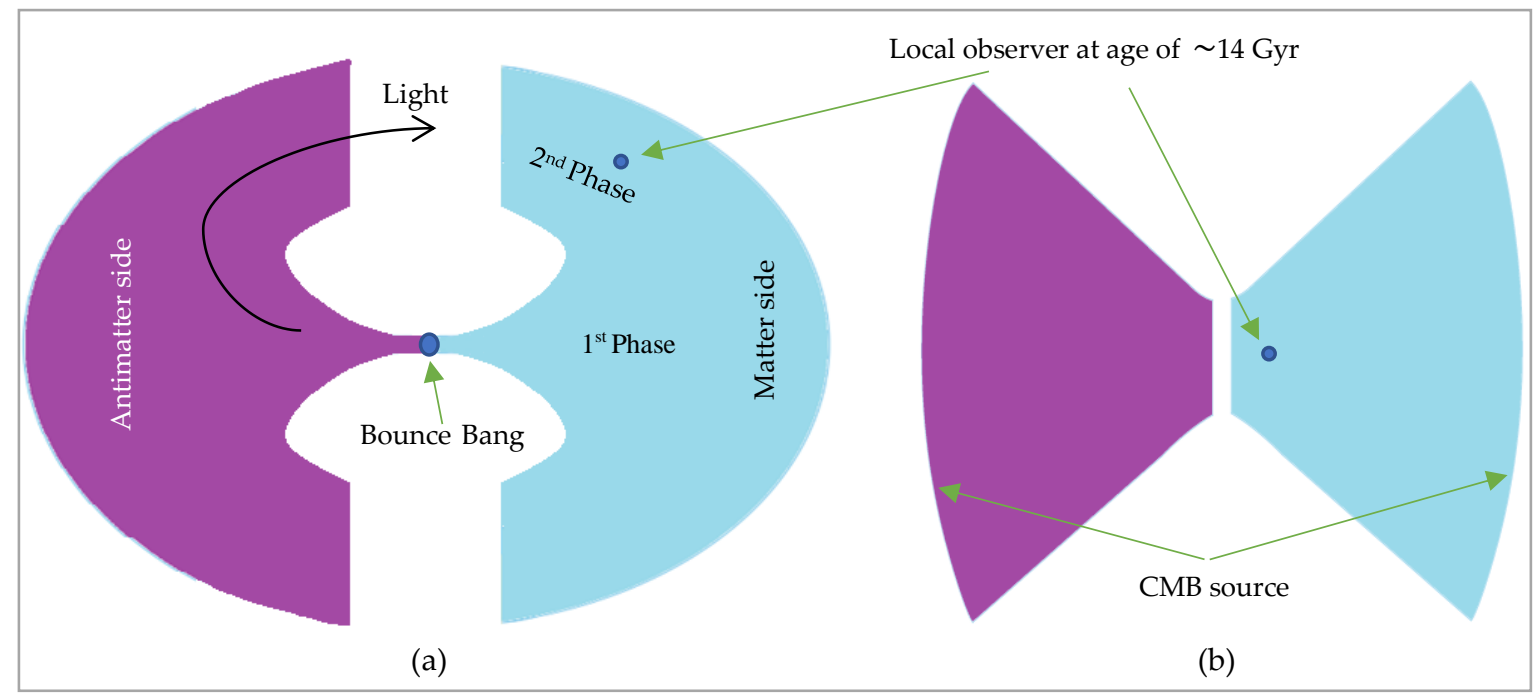

FIG. 4. (a) 2D-schematic of the predicted cosmic topology of both sides at the first phase away from the Bounce Bang; and the second phase corresponding to the reversal of the expansion direction. (b) The apparent topology during the first and second phases caused by gravitational lensing.

Light leaving antimatter side would pass to the matter side and vase versa in continued loops, which could explain why we can still observe the CMB light while we move through space much slower than light. 


\section{EXPANSION AND FLOW RATES}

\subsection{Evolution of the Hubble Parameter}

The Hubble parameter, or the speed of spatial expansion, $H$, and the acceleration $\dot{H}$ can be determined using Eqs. (15) and (13 in 8), respectively. The predicted speed and acceleration of the spatial expansion are shown in Fig. 5.

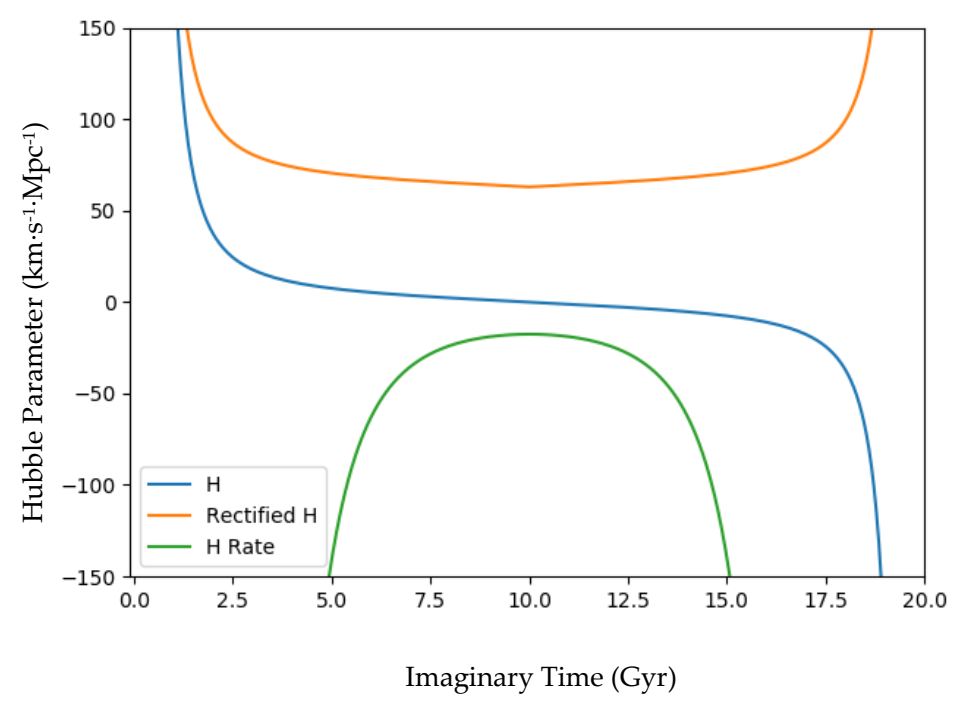

FIG. 5. Hubble parameter, or the speed of spatial expansion, and its acceleration.

The Hubble parameter, depicted by the blue curve, starts with a hyperbolic expansion/inflation at the nascent stages, perhaps owing to the Bang where the speed is at its highest value. Then, the speed of expansion decreases during the first $\sim 9 \mathrm{Gyr}$, which could be due to gravity, until it reaches its minimal value at the phase transition at $\sim 9$ Gyr. Next, the Hubble parameter starts to increase in the reverse direction (the negative sign for the speed in the second phase indicates the opposite direction, as shown in the previous sections), which is the result of both sides starting to free-fall towards each other. The parameter then reaches its zenith as it nears the Big Crunch.

According to mechanics, the opposite signs of acceleration (green curve) and expansion speed in the first phase indicate that the expansion rate is slowing down, while the matching signs in the second phase indicate that the expansion speed is increasing. A rectified Hubble parameter, indicated by the orange curve, reflect expansion in the opposite direction during the second phase, where the integration constants are tuned to regulate the mean evolution value of the parameter to $\sim 70 \mathrm{~km} \cdot \mathrm{s}$ ${ }^{1 \cdot} \mathrm{Mpc}^{-1}$.

\subsection{Flow Rate of Both Sides}

Both sides are predicted to expand away from each other during the first phase, before they freefall or flow towards each other in the second phase. The flow rate/speed of the matter side of the universe resulting from the gravitational attraction of the antimatter side can be predicted by considering the derivative of the model in Eq. (16) with respect to the imaginary conformal time as follows

$$
\underset{0<\eta \leq 2 \pi}{\overrightarrow{R_{L}}}(\eta)=\mp \frac{\rho_{0} r_{0}^{2} a_{0}^{3}}{12 E_{D}} \frac{c_{\eta}{ }^{2}}{r_{\eta}^{2}}\left(\sin ^{2} \frac{\eta}{r_{0}}+H_{\eta}^{-2} a_{0}^{-2}\left(1-\cos \frac{\eta}{r_{0}}\right)^{2}\right) \frac{1}{2} e^{i \cot H_{\eta} a_{0}\left(\sin \frac{\eta}{r_{0}}\right)\left(1-\cos \frac{\eta}{r_{0}}\right)^{-1}}
$$

Eq. (17) can be utilised regarding the dark flow observations 


\section{SIMULATION OF A SPIRAL GALAXY}

The consistent patterns of the galactic rotation curves observed using precise and independent galactic redshift data have confirmed that hydrogen clouds and outermost stars are orbiting galaxies at speeds faster than those calculated using Newtonian laws. Accordingly, the dark matter hypothesis was introduced to account for the apparently missing galactic mass and explain these fast-orbital velocities $[27,28]$. However, no evidence for the existence of dark matter, which is hypothesised to account for the majority of galactic mass, has been observed since its introduction. The failure to find dark matter led to the introduction of new theories such as modified gravity and modified Newtonian dynamics [29-33]. Several recent studies have reported that many galaxies do not contain dark matter [34]. This scenario was used to inform galaxy formation simulations using modified Newtonian dynamics without considering the effects of dark matter [35]. Therefore, it seems that there is no evidence for (or agreement on) the existence or nature of dark matter, and it may not be an essential requirement for galaxy formation.

Alternatively, because the shapes of spiral galaxies are highly similar to vortex shapes, a spiral galaxy can be modelled as a forced vortex, where external momentum is exerted on galaxies by the curvature of spacetime along its worldline evolutions. The derived model in Eq. (16) predicted that the curvature of spacetime increases along its worldline evolution, with the highest degree of curvature occurring at the phase transition, as shown in Figs. 1 and 4(a). It can therefore be inferred that the fast-orbital speeds observed for outer stars are a result of the variation of spacetime curvature along its worldline evolution.

To evaluate this inference, a fluid simulation study was performed based on Newtonian dynamics using the Fluid Pressure and Flow software [36]. In this simulation, a perfect fluid of mass density $\rho$ and isotropic pressure $p$ was assumed to represent the spacetime continuum according to the postulated spacetime duality, while the fluid particles were traced to study the divergence of fluid flux. Using these conditions, a fluid model was built to simulate the spacetime continuum flux for incrementally flatter worldline curvature, as shown in Fig. 6.

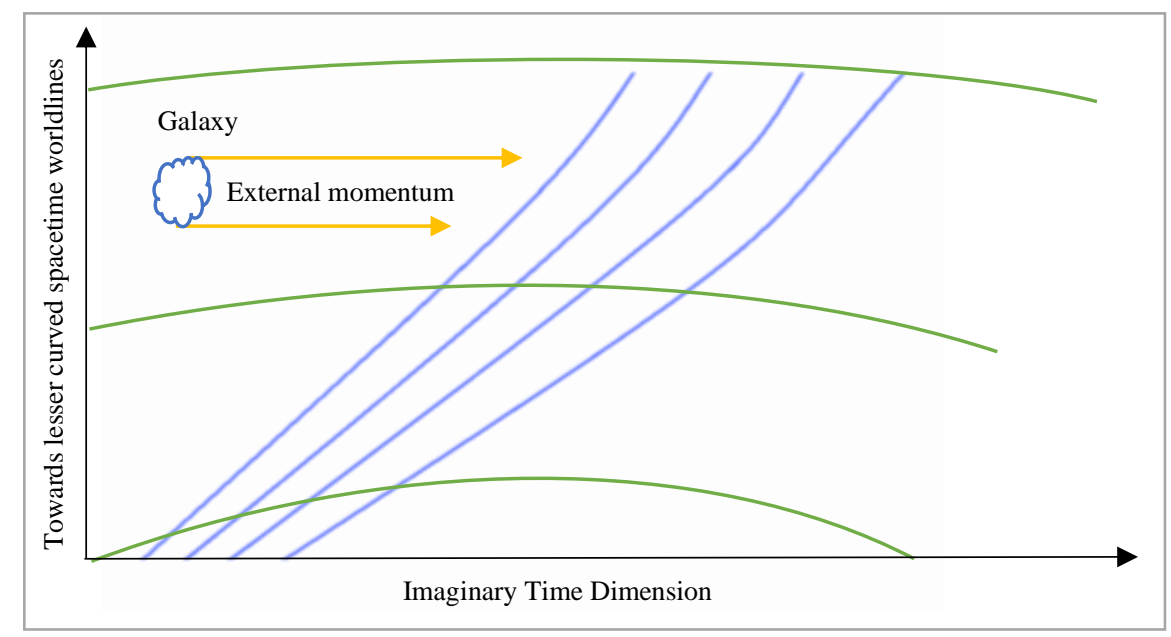

FIG. 6. External momentum exerted on a galaxy because of the divergence of spatial curvature through the imaginary time dimension along the predicted spacetime worldlines. Green curves represent the curvature of spacetime worldlines. Blue curves represent the simulated spacetime continuum flux.

Using the resultant momentum, a simulation of a spiral galaxy as a forced vortex was created, as shown in Fig. 7. 


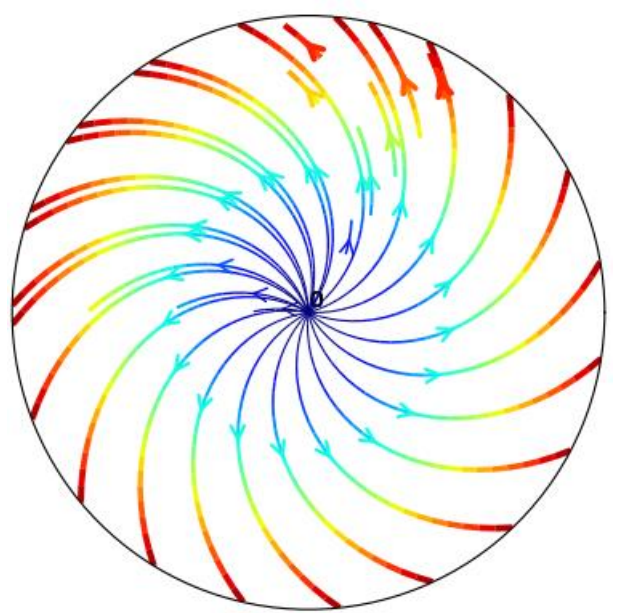

(a)

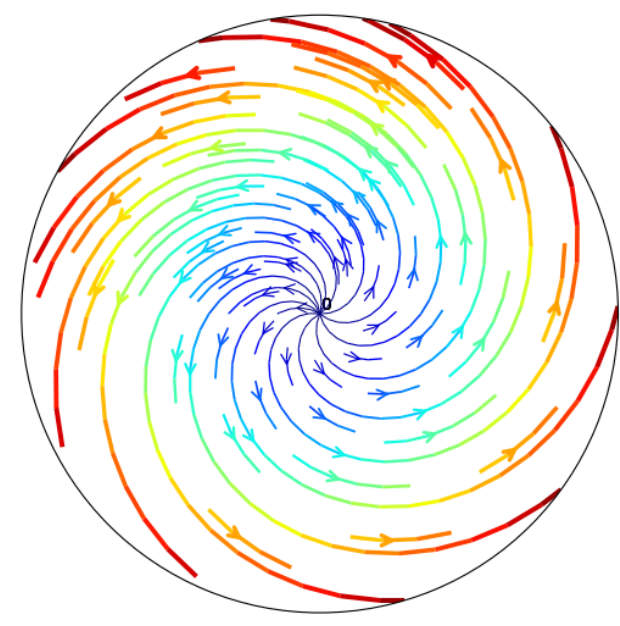

(b)

FIG. 7. Spiral galaxy rotation at (a) early and (b) present universe. Blue represents the slowest tangential speeds, and red represents the fastest speeds.

The simulation shows that the tangential speeds of outer parts of the spiral galaxy are faster in comparison with the speeds of inner parts. Additionally, the galaxies in the present universe are rotating faster than they were in the early universe for the same mass. These results seem to agree with the Tully-Fisher relation [37]. Based on the simulation results, it could be concluded that the spacetime curvature along its worldlines is responsible for the high speed of galaxies, explaining the effects corresponding to the dark matter.

\section{CONCLUSIONS}

In this study, the evolution of complex spacetime worldlines was utilised to model the universe anisotropy. The mathematical derivations of a positively curved universe governed by gravity alone revealed two opposite solutions of the worldline evolution equation, implying that the universe has two sides: matter and antimatter.

The derived model predicted that a nascent hyperbolic expansion is followed by a phase of decelerating spatial expansion during the first $\sim 9 \mathrm{Gyr}$, followed by a second phase of accelerating expansion; potentially resolving the tension in Hubble parameter measurements. Both sides of the universe expand away from the Bounce Bang during the first phase due to the Bang. Then, during the second phase, they reverse their directions and fall towards each other. It is conceivable that the matter and antimatter are free-falling towards each other with gravitational acceleration, causing the current accelerating expansion of the universe. This can be explaining the effects attributed to the dark energy. Moreover, the simulated curved spacetime during the decelerating phase was demonstrated to be flattened during the accelerating phase due to a reversal in the direction of the worldlines; explaining the current space flatness.

Regarding the fast-orbital speed of stars, the simulation provided a physical explanation by which the spatial curvature through the imaginary time dimension along the spacetime worldlines of both sides was found to exert momentum on galaxies. Therefore, geometrical spacetime curvature can be causing them to increase in speed, rather than the existence of dark matter.

Finally, the model predicted a final time-reversal phase of spatial contraction leading to the Big Crunch, thereby signifying a cyclic universe. 


\section{FUTURE WORK}

This study presented a new theory with promising outcomes and predictions, which can be verified, fine-tuned, or disproved with the aid of the astronomical data. The integration constants of this model can be fine-tuned to estimate the matter/antimatter and curvature densities accurately. Finally, the accurate age can be calculated using the derived model in future works.

\section{References}

[1] M. R. Wilczynska et al., "Four direct measurements of the fine-structure constant 13 billion years ago," Sci. Adv., vol. 6, no. 17, p. eaay9672, Apr. 2020.

[2] J. P. Uzan, "Varying constants, gravitation and cosmology," Living Reviews in Relativity, vol. 14, no. 2. Albert Einstein Institut, pp. 1-155, 29-Mar-2011.

[3] K. Migkas, G. Schellenberger, T. H. Reiprich, F. Pacaud, M. E. Ramos-Ceja, and L. Lovisari, "Probing cosmic isotropy with a new X-ray galaxy cluster sample through the L X- T scaling relation," Astron. Astrophys., vol. 636, p. 15, Apr. 2020.

[4] E. Di Valentino, A. Melchiorri, and J. Silk, "Planck evidence for a closed Universe and a possible crisis for cosmology," Nat. Astron., vol. 4, no. 2, pp. 196-203, Feb. 2020.

[5] A. TRAUTMAN, "Spin and Torsion May Avert Gravitational Singularities," Nat. Phys. Sci., vol. 242, no. 114, pp. 7-8, Mar. 1973.

[6] G. Unger and N. Popławski, "Big Bounce and Closed Universe from Spin and Torsion," Astrophys. Journal, 87078 (7pp), 2019, 2019.

[7] O. Klein, "Instead of cosmology," Nature, vol. 211, no. 5056. Nature Publishing Group, pp. 1337-1341, 1966.

[8] O. Klein, "Arguments concerning relativity and cosmology," Science (80-. )., vol. 171, no. 3969, pp. 339-345, Jan. 1971.

[9] J. Creswell, S. Von Hausegger, A. D. Jackson, H. Liu, and P. Naselsky, “On the time lags of the LIGO signals," J. Cosmol. Astropart. Phys., vol. 2017, no. 8, p. 013, Aug. 2017.

[10] E. Di Valentino, A. Melchiorri, and J. Silk, "Cosmic Discordance: Planck and luminosity distance data exclude LCDM," arXiv Prepr. arXiv2003.04935, Mar. 2020.

[11] N. Aghanim et al., "Planck 2018 results. V. CMB power spectra and likelihoods," Astron. Astrophys., vol. 89, Jun. 2020.

[12] M. Meneghetti et al., "An excess of small-scale gravitational lenses observed in galaxy clusters," Science (80-. )., vol. 369, no. 6509, pp. 1347-1351, Sep. 2020.

[13] S. Perlmutter et al., "Measurements of Omega and Lambda from 42 High-Redshift Supernovae," Astrophys. J., vol. 517, no. 2, pp. 565-586, Dec. 1998.

[14] A. G. Riess et al., "Observational Evidence from Supernovae for an Accelerating Universe and a Cosmological Constant," Astron. J., vol. 116, no. 3, pp. 1009-1038, Sep. 1998.

[15] A. G. Riess et al., "New Parallaxes of Galactic Cepheids from Spatially Scanning the Hubble Space Telescope: Implications for the Hubble Constant," Astrophys. J., vol. 855, no. 2, p. 136, Jan. 2018.

[16] A. G. Riess, "The expansion of the Universe is faster than expected," Nature Reviews Physics, vol. 2, no. 1. Springer Nature, pp. 10-12, 01-Jan-2020.

[17] T. Josset, A. Perez, and D. Sudarsky, "Dark Energy from Violation of Energy Conservation," Phys. Rev. Lett., vol. 118, no. 2, p. 021102, Jan. 2017. 
[18] E. Lusso et al., "Tension with the flat $\{\backslash$ Lambda\}CDM model from a high redshift Hubble Diagram of supernovae, quasars and gamma-ray bursts," Astron. Astrophys., vol. 628, Jul. 2019.

[19] M. Al-Fadhli, "A new vision of the geometry of the cosmos as a twin-half closed Universe: accelerated expansion due to free fall gravitational attraction," NATASTRON-20012929 Lines 18-21 submited 1400 GMT, 13/1/2020.

[20] G. Efstathiou, "Is the low cosmic microwave background quadrupole a signature of spatial curvature?," 2003.

[21] R. J. Adler, B. Casey, and O. C. Jacob, "Vacuum catastrophe: An elementary exposition of the cosmological constant problem," Am. J. Phys., vol. 63, no. 7, pp. 620-626, Jul. 1995.

[22] N. Straumann, "General Relativity (Graduate Texts in Physics)," in Springer, Springer, 2013.

[23] M. Lachì Eze-Rey and J.-P. Luminet, "COSMIC TOPOLOGY," arXivgr-qc/9605010v2 9 Jan 2003, 2003.

[24] G. F. R. Ellis and H. van Elst, “Cosmological models (Carg \’\{e\}se lectures 1998),” Dec. 1998.

[25] J. Fl, L. D. Landau, and E. Ml Lifshitz, "The Classical Theory of Fields Third Revised English Edition Course of Theoretical Physics," 1967.

[26] B. Ryden, Introduction to Cosmology. San Francisco, CA, USA: Addison Wesley, ISBN 0-80538912-1., 2006.

[27] P. D. Mannheim and D. Kazanas, "Exact vacuum solution to conformal Weyl gravity and galactic rotation curves," Astrophys. J., vol. 342, p. 635, Jul. 1989.

[28] Y. Sofue and V. Rubin, "Rotation Curves of Spiral Galaxies," Annu. Rev. Astron. Astrophys., vol. 39, no. 1, pp. 137-174, Oct. 2000.

[29] A. Maeder, "An alternative to the LCDM model: the case of scale invariance," Astrophys. J., vol. 834, no. 2, p. 194, Jan. 2017.

[30] M. M. Brouwer et al., "First test of Verlinde's theory of Emergent Gravity using Weak Gravitational Lensing measurements," Mon. Not. R. Astron. Soc., vol. 466, no. 3, pp. 2547-2559, Dec. 2016.

[31] E. A. Chadwick, T. F. Hodgkinson, and G. S. McDonald, "Gravitational theoretical development supporting MOND," Phys. Rev. D - Part. Fields, Gravit. Cosmol., vol. 88, no. 2, p. 024036, Jul. 2013.

[32] J. R. Van Meter, “Dark-matter-like solutions to Einstein's unified field equations," Phys. Rev. D, vol. 97, no. 4, p. 044018, Feb. 2018.

[33] M. Milgrom, “MOND in galaxy groups: A superior sample," Phys. Rev. D, vol. 99, no. 4, p. 044041, Feb. 2019.

[34] Q. Guo et al., "Further evidence for a population of dark-matter-deficient dwarf galaxies," Nat. Astron., vol. 4, no. 3, pp. 246-251, Jul. 2019.

[35] N. Wittenburg, P. Kroupa, and B. Famaey, "The formation of exponential disk galaxies in MOND," Astrophys. J., vol. 890, no. 2, p. 173, Feb. 2020.

[36] Sam Reid et al, "Fluid Pressure and Flow, PhET Interactive Simulations." University of Colorado, 2013.

[37] R. B. Tully and J. R. Fisher, "A new method of determining distances to galaxies," Astron. Astrophys., vol. 54, pp. 661-673, 1977. 